\title{
The referential communication skills of children with imaginary companions
}

\author{
Anna C. Roby and Evan Kidd
}

School of Psychological Sciences, The University of Manchester, UK

\begin{abstract}
The present study investigated the referential communication skills of children with imaginary companions (ICs). Twenty-two children with ICs aged between 4 and 6 years were compared to 22 children without ICs (NICs). The children were matched for age, gender, birth order, number of siblings, and parental education. All children completed the Test of Referential Communication (Camaioni, Ercolani \& Lloyd, 1995). The results showed that the children with ICs performed better than the children without ICs on the speaker component of the task. In particular, the IC children were better able to identify a specific referent to their interlocutor than were the NIC children. Furthermore, the IC children described less redundant features of the target picture than did the NIC children. The children did not differ in the listening comprehension component of the task. Overall, the results suggest that the IC children had a better understanding of their interlocutor's information requirements in conversation. The role of pretend play in the development of communicative competence is discussed in light of these results.
\end{abstract}

\section{Introduction}

Children begin to engage in large amounts of pretend play during their second year of life and continue to do so throughout childhood (Lillard, 2002, and references therein). The propensity to engage in pretend play has been linked to several important developmental milestones, including the development of self-recognition (Lewis \& Ramsay, 2004), theory of mind (Astington \& Jenkins, 1995; Nielsen \& Dissanayake, 2000; Taylor \& Carlson, 1997), and language (Bates, Benigni, Bretherton, Camaioni \& Volterra, 1979; McCune, 1995). Acts of pretence are instances of symbolic manipulation; they mark the child's burgeoning ability to engage in metarepresentational thinking (Leslie, 1987).

A group of children who by definition regularly engage in pretend play are those that have imaginary companions (ICs). Bouldin and Pratt (1999) define an IC as 'a very vivid imaginary character that does not actually exist but is treated as real by the child, who plays with it and refers to it in conversation throughout the day' (p. 400). Different researchers have categorized ICs in three ways. The first are the most commonly cited examples - invisible characters. The second are personified objects, when a child attributes animate properties to an external object (e.g. stuffed animals endowed with animate and human-like personality traits). Finally, children who engage in extended role play by adopting alternative personas have been argued to engage in processes that are qualitatively similar to having an IC (e.g. Batman) (see Taylor, 1999). Current estimates suggest that around $65 \%$ of children will have an imaginary companion within the first eight years of life (Taylor, Carlson, Maring, Gerow \& Charley, 2004). Although research on this special population has been sporadic over the last century, the results of a small number of studies suggest that having an IC confers a developmental advantage in a number of important socio-cognitive areas.

Taylor and Carlson (1997) conducted a large-scale individual differences study that investigated the relationship between the tendency to engage in fantasy play (pretence) and the development of theory of mind. Three- and 4-year-old children were tested on a range of theory of mind tasks, and their tendency to engage in fantasy play was assessed in an interview and behaviourally through a range of play-based tasks. Additionally, the children completed a test of verbal intelligence. The sample was divided into two groups based on the amount of fantasy play in which they engaged: a High Fantasy group, which consisted of children who either had an IC or often adopted a different persona, and a Low Fantasy group, which consisted of children who did not have an IC and did not regularly adopt another persona. The results showed that the 4-year-old children in the High Fantasy group performed significantly better than the children in the 4-year-old Low Fantasy group on the battery of theory of mind tasks, even when the effects of age and verbal intelligence were partialled out, suggesting that engaging in large amounts of pretence makes a unique contribution to the development of 
theory of mind. Although the direction of this effect is unclear, Taylor and Carlson argued that since both pretence and theory of mind require an ability to metareprepresent (see Leslie, 1987), and since children begin to engage in pretence prior to passing theory of mind tasks, the tendency to engage in fantasy play is likely to play a facilitative role in theory of mind development.

Taylor et al. (2004) followed up the children tested by Taylor and Carlson (1997) three years later, testing the children on aspects of their emotional understanding, self-perception, and personality. Contrary to expectations, they showed that many children still have imaginary companions or engage in extended role play after they have commenced primary school, suggesting that children's fantasy life that involves ICs and role play is just as rich as it is before they begin school. Children's ICs were not necessarily stable over this extended period, since many children had replaced old ICs with new ones. Taylor et al. also showed that the children's theory of mind scores at age 4 years significantly predicted their emotional understanding three years later, suggesting a correlational link between early fantasy play, theory of mind development, and later emotional understanding.

Bouldin, Bavin and Pratt (2002) reported that children with ICs produced a range of more complex sentence types in a narrative task than matched control children without imaginary companions. Children aged $4-8$ years were required to provide a spontaneous narrative about an imaginary monster. The authors analysed the narratives for the frequency of multiple clause sentences, which are indicative of linguistic sophistication, since they show that children can express interrelated semantic propositions together using the grammatical tools of the language. Their results showed that the IC children produced significantly more adverbial clauses (e.g. I read the newspaper while I ate), relative clauses (e.g. This is the man that eats lots of ice-cream), and compound sentences where clauses were connected by and and but (e.g. I went there but couldn't find a seat) than did the NIC children. These results supported earlier observations made by Singer and Singer (1981), who reported some evidence to suggest that having an IC aids early language development. Since children generally begin to use these complex sentence types before they are 4 years (Diessel, 2004), these data suggest that having an IC facilitates the mastery of grammatical conventions rather than their acquisition. The precise source of the advantage is unclear; however, as Bouldin et al. suggest, the IC children's practice at regulating communicative episodes with their IC could facilitate the acquisition of verbal skills because it provides them with ample opportunity to use the conventions associated with conversation.

Despite there being numerous advantages associated with having an IC, other reported differences have not replicated and are thus more contentious. Some studies have reported that IC children perform better than NIC children on tests of verbal intelligence (Mauro, 1991;
Taylor \& Carlson, 1997). However, this relationship does not seem to hold for children of all socioeconomic backgrounds. Manosevitz, Fling and Prentice (1977), for instance, showed that IC and NIC children from upper-middle-class backgrounds did not differ on tests of verbal intelligence. Other studies have reported that IC children are better able to maintain attentional focus than NIC children (Mauro, 1991; Singer, 1961), but have not been replicated in similar studies (e.g. Manosevitz et al., 1977).

These observed advantages, some of which still need corroboration, suggest that consistent and prolonged engagement in fantasy play correlates positively with many developmental milestones. Beyond the observation of this correlational fact the source of the advantage is unclear. If we assume that engaging in pretence facilitates these developmental processes, ${ }^{1}$ then there are a number of alternative explanations. Taylor (1999) suggests that through pretend play children appreciate the role of representation in mental life. Lillard (2001) argues that pretence acts as a 'twin earth' for children, where they are able to reason about relationships in the world within the safe and less restricted confines of fantasy; the more children engage in pretence the more opportunities they have to discover that, for instance, other people possess different mental states and perspectives on events. Similarly, Harris (2000) proposes that children who engage in a particular variety of pretence, role play, become more adroit and flexible thinkers because they gain more practice at simulating thought processes other than their own.

The research we have reviewed suggests that children with ICs possess socio-cognitive and linguistic advantages in relation to their same age peers. In the current paper we report on a study that aimed to extend these results to a domain where both taking another's perspective and communicative competence are important referential communication. Referential communication tasks measure children's ability to encode and decode verbal information (Lloyd, Camaioni \& Ercolani, 1995). In a typical experiment children sit opposite an interlocutor, often an experimenter. Both participants have a book of pictures in front of them. An occluding screen between the participants prevents them from seeing each other's book, and so they can only use language to convey information about the pictures in front of them. In the variant of the task that we used in our experiment, the child is required to both unambiguously describe a picture to an experimenter and appraise the informativeness of an experimenter's description of a picture, which often does not unambiguously identify a unique referent. Success on the task requires children to engage in metacognitive processing. When producing a description of a picture the child must take the perspective of the experimenter to identify the experimenter's communicative

\footnotetext{
${ }^{1}$ We distinguish this claim from pretence being a logically prior precursor to the development of these cognitive achievements, which is much more difficult to claim.
} 
needs, and encode this information into a communicative speech act. When receiving a description from the experimenter the child must evaluate the informativeness of the message and decide whether (a) the message is adequately informative, and (b) what extra information they need to successfully identify the intended picture. The task therefore requires significant cognitive and linguistic sophistication on behalf of the child.

Past research investigating the development of referential communication abilities has identified perspectivetaking and theory of mind as important contributing factors to success on the task. Roberts and Patterson (1983) identified a strong link between perspectivetaking and success on referential communication tasks. Children (aged 4-6 years) were tested on a referential communication task and two perspective-taking tasks. The first perspective-taking task assessed only the children's appreciation of their interlocutor's knowledge state; that is, whether or not their interlocutor knew the identity of the target picture before being given any descriptive information. The second task assessed the children's knowledge of the specific information that the interlocutor needed to identify the target picture. Most children performed well on the first perspective-taking task, suggesting that they understood that their interlocutor required more information to identify the correct picture. However, only the second perspective-taking task predicted success on the referential communication task; a strong positive correlation was found between these two tests, a relationship that was still strong after age was partialled out $(r=.65)$. The results suggest that although children of this age have the ability to appreciate that their interlocutor requires additional information, only those that can identify these precise informational requirements and therefore have more advanced perspective skills will be successful communicators.

In a similar vein, Resches and Pérez Pereira (2007) reported strong links between children's theory of mind performance and their referential communication abilities, where children who had the most advanced theory of mind abilities were also the most effective communicators. Astington (2003) has discussed similar results. These studies provide further evidence to suggest that sophisticated communicative abilities are dependent on perspective-taking abilities.

Since past research has shown that children with ICs perform better than children without ICs on tasks measuring socio-cognitive skill of perspective-taking (theory of mind) and language, and since perspective-taking predicts success on referential communication tasks, the current study investigated the referential communication skills of children with and without ICs. The children were aged between 4 and 6 years. Following Roberts and Patterson (1983) and Reches and Pérez Pereira (2007), this is the age range in which we are likely to see variation in performance that can be attributed to factors such as perspective-taking ability. We hypothesized that, following the results of Taylor and Carlson (1997) and
Bouldin et al. (2002), children with ICs would perform better on a referential communication task than children without ICs. Additionally, we investigated whether there were any age-related differences in the development of referential communication skills that interacted with IC status.

\section{Method}

\section{Participants}

The participants were recruited from a registry of families in the Max Planck Child Study Centre at the University of Manchester. Letters were sent to all the parents on the database who had children between the ages of 4 and 6 years, asking whether their children had an imaginary companion. Additionally, more children were recruited through media advertising. Therefore our sample was parent-selected. The control children were recruited through the Max Planck Child Study Centre, which has a large database of over one thousand families. To match each IC child we contacted the families of two to four children that could act as potential matches. If more than one family responded to our request, all children were tested, and the child whose age most closely matched the IC child was included in the final sample. Therefore, although we only report on data from our final sample, we tested approximately double the amount of NIC children. The final sample consisted of 44 children: 22 children with imaginary companions (IC) and 22 children without (NIC) ( $M=4 ; 11$ years). The IC group's age range was $3 ; 8-6 ; 5$ years $(M=4 ; 10)$ and the NIC group's was $3 ; 9-6 ; 4$ years $(M=4 ; 11)$. For the purposes of analysis each group was divided via a median split into a 'young' and an 'old' age group (for both groups $n=22 ; 11$ ICs and 11 NICs). The age range for the young group was $3 ; 8-4 ; 9$ years $(M=4 ; 3)$; the age range of the older group was $4 ; 10-6 ; 5(M=5 ; 6)$. The decision to break the children up into two age groups was motivated by the possibility of finding age-related developmental differences.

IC status was determined by means of a questionnaire given to parents, who described their children's ICs. We required the IC children to have or have recently had an IC that was a stable presence in their life for at least 6 months. The average amount of time the IC children had an IC for was 2 years (range: 6 months -4 years). Nineteen children currently had an IC and three had stopped playing with their IC in the 12 months prior to testing. The children were then asked about their IC(s), and if they corroborated the information that their parent provided they were included in the study. We required the children, as well as their parents, to provide the same name of their IC(s). One child was excluded from the sample because he did not corroborate his parent's description of his IC. We also asked the NIC children whether they had an imaginary companion; none claimed to have one, confirming their parents' report. 
Table 1 Examples of imaginary companions

\begin{tabular}{ll}
\hline Name & Description \\
\hline Imaginary family & $\begin{array}{l}\text { Imaginary ex-wife, baby that had an imaginary } \\
\text { mother who was a nurse who travelled } \\
\text { internationally, imaginary brothers. }\end{array}$ \\
$\begin{array}{ll}\text { Imaginary female dog. } \\
\text { Petsie }\end{array}$ & $\begin{array}{l}\text { An invisible girl and an imaginary dragon. } \\
\text { Bodder \& Bunn } \\
\text { Imaginary family }\end{array}$ \\
$\begin{array}{ll}\text { I don't know } & \text { An imaginary twin brother. }\end{array}$ \\
\hline
\end{tabular}

All of the IC children had either one or more invisible friends, and some $(n=2)$ had both invisible friends and personified objects. Therefore the sample contained mainly children who had only invisible friends. Our sample may have been skewed by our method of recruitment: in the first instance we relied on parents to identify whether or not their child had an IC, and the general public's understanding of what constitutes an IC may be skewed to invisible friends because they are the most common variety that appear in popular culture media such as literature, film, and television. As in past research, the children's ICs were varied and reflective of these children's very active imaginations. Table 1 provides some examples of the children's ICs.

We employed a fairly rigorous matching procedure. The IC and NIC children were matched by age, gender, birth order, number of siblings, and parental education as a proxy for socioeconomic status (SES), since this variable has been shown to significantly contribute to children's performance on referential communication tasks (Lloyd, Mann \& Peers, 1998) and linguistic development in general (e.g. Bowey, 1995; Hoff, 2003). A significant majority of children $(77 \%)$ had parents who had both completed a university undergraduate degree and/or postgraduate qualifications, $20 \%$ had one parent who had completed a university degree and another who had completed high school, and 3\% had parents who had both completed high school. To match on age we allowed a 6-month range between children with ICs and their NIC match; that is, an NIC child could be maximally 3 months younger or older than her IC match, but not outside of this range. Children were matched on birth order because this variable has been shown to be related to the likelihood of having an IC (Bouldin \& Pratt, 1999; Gleason, Sebanc \& Hartup, 2000). The children were matched on number of siblings to control for any maternal input, both linguistic and play-based, that might occur were they to have different family structures.

We did not match the children on verbal intelligence. Dixon (1982) concluded from a meta-analysis of referential communication studies that although some studies have reported low to moderate correlations between verbal intelligence and success on referential communication tasks, many studies have not, and thus referential communication tasks appear to measure a construct that is not reducible to mere vocabulary, which is the standard measure of verbal intelligence. This conclusion is supported by individual differences studies of referential communication that have shown differences between groups matched on vocabulary size (e.g. Riccio, 1998; for an extended discussion see Astington, 2003).

Each group consisted of nine only children, seven first-born children and six second-born children. In the young IC group there were five girls and six boys; in the old IC group there were six girls and five boys.

\section{Materials}

All children completed the Test of Referential Communication (ToRC) (Camaioni et al., 1995). The test comprises two identical 30-page picture books, each page either assessing the children's skills as a speaker or a listener. A barrier was placed in-between the child and experimenter to avoid the use of non-verbal forms of communication such as pointing.

During the experimental session the parents/guardians of the children completed the 17-page 'Imaginative Play Activities of Children' questionnaire, first developed by Manosevitz, Prentice and Wilson (1973) and later modified by Bouldin and Pratt (1999). The questionnaire asked parents to provide details about the children's play activities, behaviour, belief in mythical characters, and details about their imaginary companion(s), if they had any. We report on five parental ratings from the Imaginative Play Activities Questionnaire. The first four measured personality characteristics of the children, including: (i) the degree to which parents rated their children as 'outgoing, ${ }^{2}$ (ii) their ability to talk and interact with adults ('Adult interaction'), (iii) their ability to talk and interact with other children ('Child interaction'), and (iv) imaginativeness. The final measure was number of hours the children spend playing with other children when at home. We included this measure in addition to the personality measurements because we were interested in whether the time spent playing with others was related to performance on the ToRC. This is because interactions that involve negotiating activities in joint play may contribute to the development of referential communication skills, as it has been shown to be related to the development of theory of mind (Astington \& Jenkins, 1995).

\section{Procedure}

Each child was tested individually in a quiet room with only the experimenter and their parent/guardian present. They were told they would be playing a game with picture books. A practice session introduced the children to the task. Children looked at three sets of picture arrays with the experimenter. The pictures differed on certain

\footnotetext{
${ }^{2}$ Bouldin and Pratt (1999) called this dimension 'Shyness'; however, since most of the parents placed their children on the 'outgoing' end of the continuum, we chose to call it outgoing to emphasize the positive nature of these ratings.
} 
dimensions, just as the experimental items did. The experimenter explained to the child during the practice session how the pictures differed. The purpose of the practice was to make children aware that they would be required to make discriminations between sets of similar pictures. Testing began after the experimenter was confident the child understood the requirements of the task.

The ToRC has two components: a speaker component, where the child must identify a unique picture to the experimenter, and a listening comprehension component, where the child must identify a unique picture from information provided to them by the experimenter. In both conditions children are presented with an array of pictures that depict the same object or thing; for instance, a clown. Each picture varies on two or more dimensions. For example, in the item that contains four pictures of the same clown, each picture differs according to (i) the colour of the clown's collar (red or green), and (ii) the type of hat the clown is wearing (top or bobble-hat). In the speaker component, children are required to describe to the experimenter one picture that is identified by a red border. To be successful they must take into account the attributes that make it different from the other similar pictures on the same page. For instance, if required to identify the clown that has a green collar and a top hat, they must determine these characteristics as uniquely identifying the clown and use this information in their description to the experimenter. Identifying the information requires the child to compare the target picture to the other pictures by scanning the whole picture array. The listening comprehension component has two subcomponents. In the unambiguous condition, children are given sufficient information to correctly identify the picture. For instance, if required to identify the clown with the green collar and the top hat, children would be provided with both of these attributes by the experimenter. In the ambiguous condition, the children are provided with a description that can fit two or more pictures in their array. For instance, they might be told to find the picture of the clown with the green collar, which in this case would be consistent with two pictures. This subcomponent tested children's ability to identify the informativeness of the experimenter's descriptions, since if they detect the ambiguity, they should request more information. The unambiguous subcomponent serves as a baseline for the ambiguous subcomponent.

The speaker and listening components of the task are tested simultaneously within the context of a game, where sometimes it is the experimenter's turn to speak and other times it is the child's turn to speak. There are never more than two speaker or listener items in a row.

\section{Scoring}

The ToRC gets progressively more difficult; the number of pictures per page progressively increases from four to 12 , resulting in an increase in the number of distinguish- ing attributes the children must identify. The pictures systematically vary on the following dimensions: shape, colour, size, spatial location, quantity, and change of state. Overall, the ToRC contains 13 speaker trials and 17 listening comprehension trials. The maximum score for the speaker task is 35 ; more complex items (i.e. those with more pictures and more dimensions on which to compare) provide children with the opportunity to score more points. Children's score in the speaker condition is computed according to how many defining attributes they identified in the target picture. Any non-defining features described by the child were recorded as redundant features; for instance, if the child said that the clown had two buttons, which was a feature of all the clowns.

In the comprehension trials there are four unambiguous items (maximum score $=4$ ) and 13 ambiguous items. The maximum score for the ambiguous component of the comprehension trials is 60 ; once again, more complex items provide children with the opportunity to score more points because more defining features must be identified. This component is scored depending on (i) whether children's requests for more information suggest they are comparing the possible referent pictures or not, and (ii) the manner in which the children request additional information (i.e. whether they request the information in one utterance or more). For the comprehension component, any clarification questions that did not help solve uncertainty were regarded as redundant.

For the Imaginative Play Activities Questionnaire, the variables 'Outgoing', 'Child Interaction', and 'Adult Interaction' were scored on a scale from 1 to 7 , with 1 indicating, for instance, 'shy and reserved' and 7 indicating 'open and outgoing'. 'Imaginativeness' was scored on a scale from 1 to 7 , with 1 indicating 'Very imaginative' and 7 indicating 'Very unimaginative'. The number of hours children played with other children at home should be considered to be a rough estimate, since parents often indicated that they were unsure of the exact amount of time their child spent playing with others at home, and varied a lot in their estimates.

\section{Results}

\section{Imaginative Play Activities Questionnaire}

The means, standard deviations, and statistical analyses for these five measures are presented in Table 2 .

Table 2 shows that the parental ratings differed only on one dimension: Outgoing $(t(41)=2.4, p=.021, d=$ 0.75 , two-tailed). ${ }^{3}$ The parents of the IC children rated

\footnotetext{
${ }^{3}$ For $t$-tests we report Cohen's $d$, an effect size measure which signifies the magnitude of the difference between the distributions of the two means. Cohen (1992) suggests a $d=0.2$ is a small effect, $d=0.5$ is a medium effect, and $d=0.8$ is a large effect. For ANOVA results we report partial $\eta^{2}$, which reflects the amount of variance uniquely explained in the dependent variable.
} 
Table 2 Means, standard deviations, and statistical tests for the five measures analysed from the Imaginative Play Activities Questionnaire

\begin{tabular}{lccccc}
\hline IV & Group & $M$ & $S D$ & $t$ & $p^{*}$ \\
\hline Outgoing & IC & 5.66 & 1.03 & 2.4 & .021 \\
Adult Interaction & NIC & 4.73 & 1.49 & & \\
& IC & 6.37 & .774 & .11 & .913 \\
Child Interaction & NIC & 6.34 & .733 & & \\
& IC & 5.98 & .985 & .77 & .448 \\
Imaginativeness & NIC & 6.19 & .785 & & \\
& IC & 1.39 & .486 & 1.31 & .198 \\
Hours played & NIC & 1.66 & .816 & & \\
& IC & 8.05 & 8.52 & .114 & .91 \\
& NIC & 7.71 & 9.7 & & \\
\hline
\end{tabular}

* All $p$-values are two-tailed.

their children as significantly more outgoing than the parents of the NIC children.

\section{The Test of Referential Communication}

The speaker and listening comprehension components of the task were scored according to the specifications of the ToRC test manual. We report on analyses comparing the IC and NIC groups on four dependent measures: (i) speaker component score, (ii) the number of redundant features described in the speaker component of the task, (iii) listening comprehension score, and (iv) the number of questions requesting redundant information in the listening comprehension component of the task. Preliminary analyses that used gender as an independent variable yielded no significant results; therefore we did not include gender as an IV in any analyses reported in the current paper.

Table 3 presents the children's mean scores (and $S D \mathrm{~s}$ ) on the speaker component of the ToRC, and the mean number of redundant features (and $S D$ s) that children produced.

Table 3 shows that both the old and young IC groups performed better than the matched NIC groups on the speaker component of the task, and that the older children performed better than the younger children overall. The IC children also provided less redundant descriptions in the speaker task than did the matched

Table 3 Means (and SDs) for speaker component and number of redundant features in speaker task by age and IC group

\begin{tabular}{|c|c|c|c|}
\hline & Young & Old & Total \\
\hline \multicolumn{4}{|c|}{ Speaker Score } \\
\hline IC & $23.55(3.86)$ & $27.73(4.08)$ & $25.64(4.42)$ \\
\hline NIC & $18.91(4.1)$ & $26.18(4.31)$ & $22.55(5.54)$ \\
\hline Total & $21.23(4.56)$ & 26.95 (4.17) & $24.09(5.2)$ \\
\hline \multicolumn{4}{|c|}{ Redundant Descript. } \\
\hline IC & 23.09 (10.6) & $13.82(18.12)$ & $18.45(15.24)$ \\
\hline NIC & 28.09 (16.44) & $41.91(32.65)$ & $35(26.2)$ \\
\hline Total & $25.6(13.74)$ & $27.86(29.5)$ & $26.73(22.77)$ \\
\hline
\end{tabular}

NIC children, with the greatest difference being between the old IC and NIC groups.

Both speaker component dependent variables were analysed using 2 (IC group: IC versus NIC) by 2 (Age group: young versus old) univariate ANOVAs. For the speaker score there was a significant main effect of IC group $\left(F(1,40)=6.28, p=.016\right.$, partial $\left.\eta^{2}=.136\right)$, showing that the IC group performed significantly better than the NIC group overall. There was also a main effect of age group $\left(F(1,40)=21.56, p<.001\right.$, partial $\eta^{2}=$ .35 ), showing that the older children performed significantly better than the younger children. The IC group by age group interaction was not significant $(F(1,40)=$ 1.57, $p=.218$, partial $\eta^{2}=.038$ ). Although it appears that the main effect of group is carried by the large difference between the young IC and NIC groups, the non-significant interaction prevented us from comparing the children within each age group post hoc.

The redundant features data were not normally distributed, and so the data were transformed using a $\log _{10}(x+1)$ transformation for substantial positive skewness (see Tabachnick \& Fidell, 2001). A significant main effect of group showed that the IC children provided fewer redundant features than did the NIC children overall $\left(F(1,40) p=8.52, p=.006\right.$, partial $\left.\eta^{2}=.176\right)$. There was also a significant IC group by age group interaction $\left(F(1,40)=7.4, p=.01\right.$, partial $\left.\eta^{2}=.156\right)$. A simple main effects analysis showed that the older IC group produced significantly fewer redundant features than the matched older NIC group $(p<.001)$, but that the two younger groups did not differ from each other. The within-group comparisons showed that the older IC group produced significantly fewer redundant features than the young IC group $(p=.01)$, but that the NIC groups did not differ from each other. ${ }^{4}$

Table 4 presents the children's mean scores and standard deviations for the ambiguous comprehension component of the task, and the number of questions requesting redundant information in the comprehension component by age and IC group. ${ }^{5}$

\footnotetext{
${ }^{4}$ The variance in the standard deviations for the untransformed data between the groups for this analysis suggests that the assumption of homogeneity of variance has been violated. The transformation normalized the data, as indicated by a Kolmogorov-Smirnov test $(z=$ $.922, p=.363)$. However, to be sure that our results were robust we reran the analysis after deleting two outliers that deviated two standard deviations from the mean and found the same results. Additionally, we performed two non-parametric Mann-Whitney tests for independent samples, one on the young group and one on the old group. There was no significant difference in the production of redundant features for the young group $(z=.64, p=.81)$, but in the old group the NIC children produced significantly more redundant features than did the IC children $(z=2.83, p=.003$, two-tailed). From these additional analyses we can be sure that what we are observing is a robust effect. ${ }^{5}$ The children all performed well on the baseline unambiguous component of the comprehension task, with all groups scoring a mean greater than $75 \%$. A 2 (age) by 2 (IC group) ANOVA yielded no significant results. The fact that all of the children scored highly suggests that they have good language comprehension skills.
} 
Table 4 Means (and SDs) for listening comprehension score and number of redundant utterances in listening comprehension component by age and IC group

\begin{tabular}{llll}
\hline \multicolumn{1}{c}{ Young } & \multicolumn{1}{c}{ Old } & \multicolumn{1}{c}{ Total } \\
\hline \multicolumn{2}{l}{ List. Comp (Ambig.) } & & \\
IC & $9.55(6.44)$ & $20.82(14.57)$ & $15.18(12.42)$ \\
NIC & $5.73(8.1)$ & $16(12.31)$ & $10.86(11.45)$ \\
Total & $7.64(7.4)$ & $18.41(13.39)$ & $13.02(12)$ \\
Redundant & Questions & & \\
IC & $4(5.04)$ & $2.82(3.99)$ & $3.4(4.48)$ \\
NIC & $2.27(4.17)$ & $6.36(7.66)$ & $4.32(6.37)$ \\
Total & $3.14(4.6)$ & $4.59(6.23)$ & $3.86(5.46)$ \\
\hline
\end{tabular}

$N=44$.

Table 4 shows that the IC children scored higher on the ambiguous listening comprehension component of the ToRC than did the NIC children. This was true for both age groups, although the variance was high. The number of redundant questions interacted with age group and IC group: the younger IC children produced more redundant questions than did the older IC group, yet the opposite pattern was observed for the NIC children.

Both comprehension dependent variables were analysed using 2 (IC group: IC versus NIC) by 2 (Age group: young versus old) univariate ANOVAs. For the listening comprehension component there was a significant effect of age group $(F(1,40)=10.85, p=.002$, partial $\eta^{2}=.213$ ), showing that the older children performed significantly better on this component of the task than the younger children. Despite the fact that the IC children scored higher than the NIC children, the main effect for IC group was not significant $(F(1,40)=$ $1.74, p=.194$, partial $\left.\eta^{2}=.042\right)$, and neither was the age by IC group interaction $(F<1)$.

The comprehension component redundant question data were not normally distributed, and so the data were transformed using a square root transformation for mild positive skewness (see Tabachnick \& Fidell, 2001). There were no reliably significant effects, although the interaction between age group and IC group approached significance $\left(F(1,40)=3.64, p=.063\right.$, partial $\left.\eta^{2}=.084\right)$.

\section{Relationship between parental report and communicative ability}

We performed correlational analyses between the measures from the Imaginative Play Activities Questionnaire and the measures from the ToRC. The only significant result was a low but significant correlation between how outgoing the parents reported their children to be and the children's score on the speaker task $(r(43)=.309, p=$ $.022)$, a correlation that remained significant when age was partialled out $(r(43)=.34, p=.028)$. The length of time for which the IC children had possessed their IC did not correlate significantly with any of the measures collected in the ToRC.

\section{Discussion}

The IC children performed better on the speaker component of the ToRC than the NIC children, but not the comprehension component. Therefore the current data extend the range of skills in which IC children have been shown to outperform their NIC counterparts. The IC children's superior performance on the speaker component of the ToRC confirms our prediction that they would perform better than the NIC children on a test of referential communication, but is suggestive of a more subtle advantage than the children simply being better overall.

Following the results of Roberts and Patterson (1983) and Reches and Pérez Pereira (2007), who reported a strong relationship between children's performance on referential communication and higher order perspectivetaking tasks and theory of mind tasks, and Taylor and Carlson (1997), who reported that IC children pass theory of mind tasks before NIC children, we suggest that the IC children's superior performance on the speaker task derives from their increased ability to take the perspective of their interlocutor to canvass their informational requirements. This component requires the child to take into account the perspective of their interlocutor; optimum performance requires that the child must compare the target picture to the others, identify the information that makes this picture unique, and encode this information into an intelligible verbal message. The results suggest that the IC children were better than the NIC children at identifying this information and conveying it to the experimenter.

Whereas the greatest difference between age groups for the speaker score was observed in the younger age group, the greatest difference in the production of redundant utterances was observed between the older groups. We suggest that these results reflect the underlying developmental trajectory of referential communication skills. The NIC group showed the greatest improvement in the speaker score, whereas the IC group showed the greatest improvement in the production of redundant utterances. In the task, if a child successfully identifies the uniquely identifying features of the target picture to the interlocutor but provides many redundant descriptions, it suggests that the child has used a 'local' strategy in the task. That is, the child may have merely described the target picture without comparing it to the other pictures. Fewer redundant descriptions suggest that the child has used a more 'global' strategy; that is, the child has compared the target picture to the others and has identified the target's unique attributes. The fact that the young NIC group were only successful in identifying the correct picture in the speaker task just over half of the time, and that the old NIC group produced many redundant utterances, suggests that the children in the NIC group were only beginning to become aware of the requirements of the task; that is, the need to provide sufficient information for effective communication. In contrast, 
the fact that the IC groups performed well on the speaker task and showed a decrease in redundant utterances across age suggests that these children were more aware of the need to provide uniquely identifying information to their interlocutor. That is to say, the IC children better understood that the experimenter required specific information that they were then able to convey. How might having an IC confer such an advantage?

Taylor and Carlson (1997) suggested that engaging in large amounts of pretend play, as IC children do, leads to the earlier onset of theory of mind because the domain of fantasy allows children to contrive the world as it is not; that is, it provides children with an opportunity to appreciate that there can be a mismatch between mental representation and reality. Understanding that there is not always a one-to-one relationship between reality and mental representation is essential for the types of real world communicative acts the referential communication task mimics, where it is important to successfully implement conversational implicatures like the Gricean Maxims of Manner (avoid ambiguity) and Quantity (make your contribution as informative as is required, but not more so) (Grice, 1975). However, it is still unclear just what aspects of having an IC confer this advantage. Astington and Jenkins (1995) identified two aspects of pretend play that correlated significantly with successful performance on theory of mind tasks: (1) making joint proposals in pretend play (e.g. 'Let's make a mud pie'), and (2) providing explicit role assignment to self and others (e.g. 'I'll be the mummy and you be the baby'). These are cooperative features of play that generally involve other children. However, many of the measures Taylor and Carlson (1997) used involved solitary play activities that may or may not involve other children. It is possible that the IC advantage derives from children engaging in the kinds of behaviour described by Astington and Jenkins, but alone with their IC. In order to engage in a satisfactory play episode with an IC, the child must (a) define the parameters of the episode, (b) assign roles to the IC and to self, (c) actively construct a series of events or situations, and (d) construct a dialogue for all participants. The suggestion is that the advantage derives not simply from engaging in these behaviours, but from actively constructing the fantasy. In doing so children must monitor and continually update their model of the other (imaginary) participants' mind states, and on the basis of these invent conversation. Hence we see advantages in socio-cognitive functioning, as shown by Taylor and Carlson (1997), and language, as shown by Bouldin et al. (2002). This is not something that occurs when one is playing with real friends, who bring to the play episode their own unobservable (and uncontrollable) mind states and speech.

This interpretation, although speculative, argues that knowledge is acquired through action. It is consistent with the proposals made by Taylor (1999), Lillard (2001), and Harris (2000), all of whom discuss the manner in which pretence may affect development. Addition- ally, we suggest that our data provide further evidence that adds to these proposals. Taylor (1999) argued that engaging in pretence aids socio-cognitive development because this style of play allows children to engage in activities that allow them to discover the mental lives of others. Harris and Lillard, on the other hand, argue that children do not necessarily reflect on these relationships as metarepresentational. Success on referential communication tasks requires a good deal of meta-cognitive functioning; therefore we suggest that the IC children in the present study were more meta-cognitively advanced than the NIC children. If this is the case, then it would seem to support the arguments made by Taylor (1999). However, we have also argued that the children gain this advantage through actively constructing pretence, which clearly involves simulation. Therefore we suggest a key role for simulation as a methodology for pretence, but that an outcome of this type of play is an increased ability to engage in meta-cognitive processing.

Two other aspects of our data may further narrow down the source of the IC advantage. First, in comparison to other studies, our IC sample was fairly homogeneous, containing only children who had invisible companions. Gleason et al. (2000) reported that children's relationships with their invisible friends were largely describable as sociable and friendly, whereas children with personified objects tended to take a nurturing role. Therefore it is possible that our results are only applicable to children with invisible friends, derived from the nature of the relationship these children have with their ICs. Future research needs to systematically study the effect that these children's exact relationship with their IC has on their cognitive development. Second, we did not observe a significant correlation between the length of time the children had an IC and their performance on the ToRC. ${ }^{6}$ This could suggest that the children in our sample simply benefited from having an IC, irrespective of the amount of time they had one for. However, future research should investigate this relationship more systematically, since our null result could be the result of our small sample size.

Two more results warrant discussion. The first concerns the fact that we only observed a difference between the two groups on the speaker component of the task. Although performance on the two components of the task correlates $(r=.68, d f=44, p<.001)$, there was much more variability in the children's performance on the listener component compared to their performance on the speaker component. In general, young children tend to do better on the speaker component of the task than on the listener component, which suggests that the listener component requires a level of functioning

\footnotetext{
${ }^{6}$ The correlation between the length of time the children had had an IC and the number of redundant features the children provided in the speaker task approached significance $(r=-.348, d f=22, p=.056)$; however, this tendency weakened considerably once age was partialled out $(r=-.24, d f=22, p=.15)$.
} 
children of this age have yet to achieve (Lloyd et al., 1998). It seems that children at this age are not very good at evaluating the informativeness of a statement provided by an adult interlocutor. This may have been further compounded by the fact that the experimenter was unfamiliar to the children. Future studies could compare older children with and without ICs to see if the two groups differed on tasks such as message appraisal.

Second, the results from the Imaginative Play Activities Questionnaire showed that the parents of the IC children rated their children as significantly more outgoing than the parents of the NIC children. Interestingly, the parents did not differ on how imaginative they believed their child to be, despite the fact that, by virtue of having an IC, the naive assumption is that these children would be on average more imaginative. These results differ from those reported by Bouldin and Pratt (1999), who showed that IC and NIC children did not differ in how outgoing they were rated to be, but did differ in the extent to which they were rated as imaginative, where IC children were rated to be more imaginative than NIC children. Bouldin and Pratt, however, tested a much larger sample size $(N=478$, including 81 IC children $)$ over a wider age range $(2 ; 2-9 ; 5)$. Therefore the differences between the two samples could be due to a number of reasons, including the larger age range tested by Bouldin and Pratt.

Finally, we end the paper with two caveats. The first concerns the correlational nature of our data. We have attributed the superior performance of the IC children in our study to their tendency to engage in greater amounts of specific types of pretend play. However, just as in Taylor and Carlson (1997), our data are correlational, and despite careful participant matching we cannot rule out the possibility that the metarepresentational skills underlying referential communication lead children to engage in larger amounts of pretence and to the development of ICs. Nevertheless, like Taylor and Carlson, we argue that this explanation of the data is unlikely, since children begin to engage in pretence long before they engage in the advanced socio-cognitive functioning required in referential communication. Longitudinal data are required to resolve this issue.

The second caveat concerns the contribution of verbal IQ to performance on referential communication tasks. In particular, because we did not control for verbal IQ it is possible that the IC children performed better because they were more linguistically advanced. Given that Bouldin et al. (2002) showed that IC children produced more complex language than NIC children, this is a possibility. However, there are several reasons why such an explanation cannot fully account for our data. First, as argued by Dixon (1982), evidence for a relationship between verbal intelligence and performance on referential communication tasks is at best weak. Second, whether or not IC children have higher verbal ability is not clear, since although some studies have reported a difference (e.g. Taylor \& Carlson, 1997), others have only reported the difference for subsets of this population (Manosevitz et al., 1977), and others have reported no difference at all (Taylor et al., 2004).

Therefore, although we cannot fully dismiss a role for verbal intelligence in our results, we argue that the observed difference between the two groups is not totally reducible to this factor. Instead we have argued that the IC children in the present study outperformed the NIC children on the speaker component of the ToRC because they possess more advanced perspective-taking abilities. The results therefore suggest a number of different avenues for research. The first concerns the contribution of predisposition to fantasy to the development of pragmatic knowledge. The second is more general, and concerns the contribution of predisposition to fantasy to the development of meta-cognitive processing skills. Carlson and Davis (2005) reported that children's tendency to engage in representational and social pretence significantly predicted their performance on tasks measuring executive function, suggesting that IC children might have better executive control than NIC peers. Exploring where a predisposition to fantasy does and does not contribute to socio-cognitive development should increase our knowledge of the role pretence plays in development, and more importantly shed light on the possible mechanisms underlying the developmental achievements that correlate with this style of play.

\section{Acknowledgements}

This study formed part of the first author's MSc thesis. The inspiration for the study came from discussions with staff and students in the Language Development and Reading Laboratories at La Trobe University. We would like to thank the children and parents who participated in this study, Peter Lloyd and Paula Bouldin for generously providing us with materials, and audiences at the psychology departments at the Universities of Edinburgh and Oxford, and the Max Planck Institute for Evolutionary Anthropology for helpful comments.

\section{References}

Astington, J.W. (2003). Sometimes necessary, never sufficient: false-belief understanding and social competence. In B. Repacholi \& V. Slaughter (Eds.), Individual differences in theory of mind: Implications for typical and atypical development (pp. 13-38). Hove: Psychology Press.

Astington, J.W., \& Jenkins, J.M. (1995). Theory of mind development and social understanding. Cognition and Emotion, 9, 151-165.

Bates, E., Benigni, L., Bretherton, I., Camaioni, L., \& Volterra, V. (1979). Cognition and communication from 9-13 months: correlational findings. In E. Bates (Ed.), The emergence of symbols: Cognition and communication in infancy (pp. 69-140). New York: Academic Press. 
Bouldin, P., Bavin, E.L., \& Pratt, C. (2002). An investigation of the verbal abilities of children with imaginary companions. First Language, 22, 249-264.

Bouldin, P., \& Pratt, C. (1999). Characteristics of preschool and school-age children with imaginary companions. Journal of Genetic Psychology, 160, 397-410.

Bowey, J.A. (1995). Socioeconomic status differences in preschool phonological sensitivity and first-grade reading achievement. Journal of Education Psychology, 87, 476487.

Camaioni, L., Ercolani, P., \& Lloyd, P. (1995). Prova di communicazione referenziale. Florence: Organizzazioni Speciali.

Carlson, S.M., \& Davis, A.C. (May, 2005). Executive function and pretense in preschool children. Poster presented at the 35 th annual conference of the Jean Piaget Society, Vancouver, Canada.

Cohen, J. (1992). A power primer. Psychological Bulletin, 112, 155-159.

Diessel, H. (2004). The acquisition of complex sentences. Cambridge: Cambridge University Press.

Dixon, W.P. (1982). Two decades of referential communication research: a review and meta-analysis. In C.J. Brainerd \& M. Pressley (Eds.), Verbal processes in children: Progress in cognitive development research (pp. 1-33). Berlin: Springer-Verlag.

Gleason, T.R., Sebanc, A.M., \& Hartup, W.W. (2000). Imaginary companions of preschool children. Developmental Psychology, 36, 419-428.

Grice, H.P. (1975). Logic and conversation. In P. Cole \& J. Morgan (Eds.), Syntax and semantics (Vol. 3, pp. 41-58). New York: Academic Press.

Harris, P. (2000). The work of the imagination. Oxford: Blackwell.

Hoff, E. (2003). The specificity of environmental influence: socioeconomic status affects early vocabulary development via maternal speech. Child Development, 74, 1368-1378.

Leslie, A.M. (1987). Pretense and representation: the origins of 'theory of mind'. Psychological Review, 94, 412-426.

Lewis, M., \& Ramsay, D. (2004). Development of selfrecognition, personal pronoun use, and pretend play during the 2nd year. Child Development, 75, 1821-1831.

Lillard, A.S. (2001). Pretend play as Twin Earth: a socialcognitive analysis. Developmental Review, 21, 495-531.

Lillard, A.S. (2002). Pretend play and cognitive development. In U. Goswami (Ed.), Handbook of cognitive development (pp. 188-205). London: Blackwell.

Lloyd, P., Camaioni, L., \& Ercolani, P. (1995). Assessing referential communication skills in the primary school years: a comparative study. British Journal of Developmental Psychology, 13, 13-29.

Lloyd, P., Mann, S., \& Peers, I. (1998). The growth of speaker and listener skills from five to eleven years. First Language, 18, 81-103.

McCune, L. (1995). A normative study of representational play at the transition to language. Developmental Psychology, 31, 198-206.

Manosevitz, M., Fling, S., \& Prentice, N.M. (1977). Imaginary companions in young children: relationships with intelligence, creativity, and waiting ability. Journal of Child Psychology and Psychiatry, 18, 73-79.

Manosevitz, M., Prentice, N.M., \& Wilson, F. (1973). Individual and family correlates of imaginary companions in preschool children. Developmental Psychology, 8, 72-79.

Mauro, J. (1991). The friend that only I can see: a longitudinal investigation of children's imaginary companions. Unpublished doctoral dissertation, University of Oregon.

Nielsen, M., \& Dissanayake, C. (2000). An investigation of pretend play, mental state terms, and false belief understanding: in search of a metarepresentational link. British Journal of Developmental Psychology, 18, 609-624.

Reches, M., \& Pérez Pereira, M. (2007). Referential communication abilities and theory of mind development in preschool children. Journal of Child Language, 34, 21-52.

Riccio, M. (1998). Referential communication ability and specific learning disorders: comparison of children with specific learning disorders and normal children of school age. Psicologia Clinica dello Sviluppo, 2, 271-302.

Roberts, R.J., \& Patterson, C.J. (1983). Perspective taking and referential communication: the question of correspondence reconsidered. Child Development, 54, 1005-1014.

Singer, J.L. (1961). Imagination and waiting ability in young children. Journal of Personality, 29, 396-413.

Singer, J.L., \& Singer, D.G. (1981). Television, imagination, and aggression: A study of preschoolers. Hillsdale, NJ: Lawrence Erlbaum.

Tabachnick, B., \& Fidell, L. (2001). Using multivariate statistics (4th edn.). Boston, MA: Allyn \& Bacon.

Taylor, M. (1999). Imaginary companions and the children who create them. Oxford: Oxford University Press.

Taylor, M., \& Carlson, S. (1997). The relation between individual differences in fantasy and theory of mind. Child Development, 68, 436-455.

Taylor, M., Carlson, S., Maring, B., Gerow, L., \& Charley, C. (2004). The characteristics and correlates of fantasy in school-age children: imaginary companions, impersonation, and social understanding. Developmental Psychology, 40, 1173-1187.

Received: 17 July 2006

Accepted: 4 July 2007 